\section{Dementia \\ and Geriatric}

Cognitive Disorders

\title{
Qualitative Assessment of Verbal Fluency Performance in Frontotemporal Dementia
}

\author{
Esther van den Berg ${ }^{a} \quad$ Lize C. Jiskoot $^{a, b}$ Mariëlle J.H. Grosveld ${ }^{a}$ \\ John C. van Swieten ${ }^{a, c}$ Janne M. Papma ${ }^{a}$ \\ ${ }^{a}$ Alzheimer Center and Department of Neurology, Erasmus University Medical Center, \\ Rotterdam, b Department of Radiology, Leiden University Medical Center, Leiden, and \\ 'Department of Clinical Genetics, VU University Medical Center, Amsterdam, \\ The Netherlands
}

\section{Keywords}

Primary progressive aphasia $\cdot$ Executive control $\cdot$ Semantic memory $\cdot$ Dementia

\begin{abstract}
Background/Aims: Verbal fluency is impaired in patients with frontotemporal dementia (FTD) and primary progressive aphasia (PPA). This study explored qualitative differences in verbal fluency (clustering of words, switching between strategies) between FTD and PPA variants. Methods: Twenty-nine patients with behavioral variant FTD (bvFTD) and 50 with PPA (13 nonfluent/agrammatic, 14 semantic, and 23 logopenic) performed a semantic and letter fluency task. Clustering (number of multiword strings) and switching (number of transitions between clustered and nonclustered words) were recorded by two independent raters. Between-group differences, associations with memory, language, and executive functioning, and longitudinal change (subsample) in clustering and switching were examined. Results: Interrater reliability was high (median 0.98). PPA patients generated (a) smaller (number of) clusters on semantic and letter fluency than bvFTD patients $(p<0.05)$. Semantic variant patients used more switches than nonfluent/agrammatic or logopenic variant patients $(p<0.05)$. Clustering in semantic fluency was significantly associated with memory and language (range standardized regression coefficients $0.24-0.38$ ). Switching in letter fluency was associated with executive functioning (0.32-0.35). Conclusion: Clustering and switching in verbal fluency differed between patients with subtypes of FTD and PPA. Qualitative aspects of verbal fluency provide additional information on verbal ability and executive control which can be used for clinically diagnostic purposes.


van den Berg et al.: Qualitative Assessment of Verbal Fluency Performance in Frontotemporal Dementia

\section{Introduction}

Frontotemporal dementia (FTD) is an early-onset dementia characterized by a heterogeneous clinical presentation including behavioral changes, frontal executive deficits, and/or selective language disorders [1] caused by pathophysiological changes in the frontal and temporal lobes [2,3]. Patients with the behavioral variant FTD (bvFTD) typically present with personality and behavioral changes such as inappropriate social conduct, apathy, or disinhibition $[4,5]$. The language variants are collectively referred to as primary progressive aphasia (PPA) and include a semantic variant (characterized by multimodal loss of semantic knowledge), a nonfluent/agrammatic variant (characterized by agrammatism and apraxia of speech), and the more recently defined logopenic variant (characterized by a slowed rate of speech and impaired sentence repetition), which is also associated with Alzheimer-type pathology $[1,6]$.

The clinical diagnosis of FTD and PPA is aided by detailed neuropsychological examination including measures of language, executive functioning, and memory. Executive control is often evaluated by tests of verbal fluency $[7,8]$. Verbal fluency refers to the ability to generate words from a semantic category (e.g., animals, groceries, fruits) or a specified letter of the alphabet within a limited time. Verbal fluency tasks are easy to administer, and the number of correct responses provides a rich source of information on semantic, lexical, and executive cognitive processes [9]. Based on the differences in clinical subtypes of FTD and PPA, a disparate pattern of impairment in verbal fluency tasks is expected. Indeed, a previous study showed that patients with semantic variant PPA were more impaired on semantic than letter fluency, whereas patients with bvFTD and nonfluent/agrammatic variant PPA were equally impaired in both types of tasks [8].

In this study, we not only aim to examine quantitative differences in verbal fluency in FTD and PPA, but also focus on potential qualitative differences. Qualitative aspects of lexical retrieval, such as the clustering of related words and switching between categories, can offer additional insights into the cognitive processes of fluency task performance in specific patient groups, especially into the decay of semantic knowledge [10] and executive control [11]. The diagnostic utility of clustering and switching in verbal fluency has been shown for different patient groups such as those with traumatic brain injury [12] and schizophrenia [13], but it is unknown for FTD. Also, whether measures of clustering and switching provide additional information on related cognitive functions such as memory, language, processing speed, or executive functioning in these patients is unclear. Qualitative verbal fluency measures may have prognostic value, already demonstrated in the prediction of conversion from mild cognitive impairment (MCI) to Alzheimer disease (AD) [14], but whether disease progression in FTD could be predicted by clustering and switching measures remains to be evaluated.

The present study thus aimed to examine clustering and switching in semantic and letter fluency in patients with bvFTD and those with PPA. Additionally, associations with other cognitive functions, as well as longitudinal changes in fluency measures in a subsample of patients, were evaluated.

\section{Subjects and Methods}

\section{Participants}

This retrospective study included 79 patients who visited the Alzheimer Center of the Erasmus University Medical Center, Rotterdam, The Netherlands, between January 2012 and December 2015 and were diagnosed with bvFTD or PPA. The clinical diagnosis was made in a multidisciplinary consensus meeting using international diagnostic consensus criteria. For bvFTD $(n=29)$, the diagnostic criteria of Rascovsky et al. [5] were used. The diagnosis of patients with PPA (14 semantic, 13 nonfluent/agrammatic, and 23 logo- 
van den Berg et al.: Qualitative Assessment of Verbal Fluency Performance in Frontotemporal Dementia

penic) was based on the diagnostic criteria of Gorno-Tempini et al. [6]. As part of their diagnostic workup, all patients underwent a standardized clinical assessment including medical history, informant-based history, physical and neurological examinations, neuropsychological assessment (including the Mini-Mental State Examination [MMSE] and Frontal Assessment Battery [FAB]), laboratory tests, and brain imaging. Nineteen patients of the present sample ( 3 with bvFTD, 6 semantic, 4 nonfluent/agrammatic, and 6 logopenic) performed a second neuropsychological assessment at a follow-up consultation after an average of $14.9 \pm 6.0$ months. Duration of illness was defined as the interval between patient and/or informant-reported first symptoms and the first memory clinic evaluation. Level of education was classified according to the system of Verhage [15], ranging from 1 (less than primary school) to 7 (university degree).

The study was approved by the Medical and Ethics Review Committee of the Erasmus University Medical Center. The participants gave written informed consent.

\section{Verbal Fluency Measures}

All participants performed a semantic and letter fluency task as part of a standardized neuropsychological assessment (see below). For the semantic fluency task, the participants were asked to generate as many exemplars as possible from the category of animals in $60 \mathrm{~s}$. For the letter fluency task, the participants were asked to generate as many different words as possible beginning with the letter $D$, then $A$, and then $T$, with $60 \mathrm{~s}$ allowed for each letter. The letters $D, A$, and $T$ are considered the Dutch equivalent to the letters $F$, $A$, and $S$ [16]. At follow-up, the letters $K, O$, and $M$ or $P, G$, and $R$ were used as parallel versions. Participants were instructed not to generate proper names or a previously generated word with only a different suffix. The total number of correct animals and the total number of correct words generated for the letters $D, A$, and $T$ were recorded. Impaired performance of individual patients was defined as a T-score $\leq 27$ compared to age-, sex-, and education-adjusted normative data (corresponding to $\leq 1$ st percentile). In addition, the semantic and letter fluency performance of each participant was reevaluated by means of the scoring system reported by Ledoux et al. [17; adaptation of 11]. Table 1 shows a summary of the scoring guidelines; further details on the scoring procedure for clustering and switching are reported elsewhere [17]. Based on this scoring procedure, the following measures were scored for the semantic and letter fluency performance of each participant ${ }^{1}$ :

(1) Total number of words: sum of all words produced, excluding repetitions and rule breaks; for letter fluency, the sum of the letters $D, A$, and $T$ was used

(2) Number of clusters: number of multiword strings; each cluster contained at least 2 successive words

(3) Number of switches: number of transitions between clustered or nonclustered words (i.e., switches from one associative strategy to another or to none at all)

(4) Total cluster size: sum of all clustered words

(5) Mean cluster size: total cluster size divided by the number of clusters

\section{Other Cognitive Functions}

The standardized neuropsychological assessment included validated tests covering the major cognitive domains. Memory was assessed with the Dutch version of the Rey Auditory Verbal Learning Test (RAVLT [18]; immediate recall, delayed recall, delayed recognition), the story recall subtest from the Rivermead Behavioural Memory Test [19], and the visual association test [20]. Language was examined with the 60-item Boston Naming Test [21]. Information processing speed was assessed with the letter digit substitution test [22], the trail-making test part A [23], and the Stroop Color and Word Tests I and II [24]. Attention and executive functioning were assessed with the trail-making test B/A ratio, the Stroop Color and Word Test III/II ratio, the modified Wisconsin Card Sorting Test (mWCST [25]; number of concepts), the digit span subtest of the Wechsler Adult Intelligence Scale, ed 3 (WAIS-III [26]; total score), and the zoo map and key search subtests of the Behavioural Assessment of the Dysexecutive Syndrome (BADS [27]). Raw test scores were converted to standardized $z$-scores based on the mean and standard deviation of the study population. The $z$-scores were then averaged per cognitive domain into a composite domain score.

\section{Statistical Analysis}

Statistical analyses were performed using SPSS Statistics 21.0 (IBM Corp., Armonk, NY, USA). Betweengroup differences were analyzed with analysis of variance for continuous data, Mann-Whitney $U$ tests for

1 The original variable "percentage of clustered words (including errors)" yielded no meaningful data in our patient sample due to the relatively large number of errors, and was therefore omitted from the analysis. 
Dementia

and Geriatric

Cognitive Disorders

\begin{tabular}{l|l}
\hline Dement Geriatr Cogn Disord 2017;44:35-44 \\
\hline DOI: 10.1159/000477538 & $\begin{array}{l}\text { C 2017 The Author(s). Published by S. Karger AG, Basel } \\
\text { www.karger.com/dem }\end{array}$ \\
\hline
\end{tabular}

van den Berg et al.: Qualitative Assessment of Verbal Fluency Performance in Frontotemporal Dementia

Table 1. Scoring guidelines for qualitative fluency variables

\begin{tabular}{|c|c|}
\hline Successive words are considered a cluster when there are... & Example \\
\hline \multicolumn{2}{|l|}{ Semantic fluency } \\
\hline ...words that belong to the same subcategory & dog, cat, hamster \\
\hline ...words that have an obviously strong association & lion, tiger, bear \\
\hline ...words that share the same first sound & cat, kangaroo \\
\hline ...items that contain the same word & lion, sea lion \\
\hline ...words that rhyme & dog, frog \\
\hline \multicolumn{2}{|l|}{ Letter fluency } \\
\hline ...words with the same first 2 letters & snail, snow \\
\hline ...words with the same first and last sound, differing only & \\
\hline in a vowel sound & simple, sample \\
\hline ...words that are homophones & see, sea \\
\hline ...words that rhyme & smart, start \\
\hline ...words with semantic/associative relationships & sneaker, sock; salt, shaker; sit, stand \\
\hline
\end{tabular}

ordinal data, and $\chi^{2}$ tests for dichotomous data. All analyses were adjusted for age and sex. Analyses of the qualitative fluency variables were additionally adjusted for the total number of words generated. Because the number of patients with PPA was limited, the primary analysis of the fluency variables examined the difference between the bvFTD group and the three PPA groups (semantic, nonfluent/agrammatic, and logopenic variants) taken together by means of a one-way univariate analysis of covariance. In case of a significant difference in the primary analysis, post hoc comparisons between the PPA groups were performed. Interrater reliability was calculated by means of intraclass correlation analysis. Change over time for the fluency variables was performed by means of repeated-measures analysis of variance, including main effects of "time" and "group" and the "time $\times$ group" interaction. Associations between fluency variables and composite cognitive domains were examined with stepwise regression analysis, including age and sex in step 1 and the composite cognitive domain scores (language, memory, processing speed, attention, and executive functioning) in step 2 . The $\alpha$ value was set at 0.05 (two-tailed) for all comparisons.

\section{Results}

\section{Demographics}

The characteristics of the patient sample are presented in Table 2 . The subgroups differed significantly in age $\left(F(3,75)=3.6, p<0.05, \eta^{2}=0.13\right)$ and gender $\left(\chi^{2}(3)=12.4, p<0.01\right)$, with higher age and more women in the logopenic group than in the other groups. The groups did not differ in educational level, disease duration, or FAB score (all $p>0.05$ ), but the logopenic group had a lower MMSE score than the bvFTD and the nonfluent/agrammatic variant patients $\left(F(3,71)=6.5, p<0.01, \eta^{2}=0.22\right)$.

\section{Interrater Reliability}

Two independent raters, blinded to patient status, scored the fluency clustering and switching parameters (see Subjects and Methods) based on the scoring system described in Ledoux et al. [17]. Table 3 shows high levels of interrater reliability for both the semantic (range 0.90-1.0) and the letter fluency variables (range 0.80-0.99). 
van den Berg et al.: Qualitative Assessment of Verbal Fluency Performance in Frontotemporal Dementia

Table 2. Demographics, disease characteristics, and verbal fluency performance in patients with bvFTD and those with PPA

\begin{tabular}{|c|c|c|c|c|c|}
\hline & \multirow[t]{2}{*}{ bvFTD } & \multicolumn{3}{|l|}{ PPA } & \multirow[t]{2}{*}{ Statistics } \\
\hline & & SD & PNFA & LPA & \\
\hline Subjects, $n$ & 29 & 14 & 13 & 23 & ns \\
\hline Age, years & $58.9 \pm 9.4$ & $65.8 \pm 9.1$ & $64.0 \pm 12.0$ & $67.0 \pm 8.3$ & $p<0.05$ \\
\hline Sex, $n$ (\% male $)$ & 20 (69) & $8(57)$ & $8(62)$ & $5(22)$ & $p<0.05$ \\
\hline Level of education, score & $4.6 \pm 1.2$ & $4.9 \pm 1.4$ & $4.9 \pm 1.6$ & $4.4 \pm 1.4$ & ns \\
\hline MMSE score & $25.3 \pm 4.0$ & $25.0 \pm 2.9$ & $27.7 \pm 2.2$ & $21.9 \pm 4.3$ & $p<0.05$ \\
\hline Frontal Assessment Battery score & $13.3 \pm 3.9$ & $13.7 \pm 2.8$ & $13.9 \pm 2.8$ & $11.6 \pm 3.1$ & ns \\
\hline Symptom onset, years & $3.6 \pm 3.0$ & $3.6 \pm 1.6$ & $2.8 \pm 1.0$ & $2.6 \pm 1.6$ & ns \\
\hline \multicolumn{6}{|l|}{ Measures of verbal fluency ${ }^{\mathrm{a}}$} \\
\hline \multicolumn{6}{|l|}{ Semantic fluency } \\
\hline Number of words & $13.5 \pm 6.3$ & $8.2 \pm 4.9$ & $10.5 \pm 5.7$ & $10.4 \pm 5.0$ & $p<0.05$ \\
\hline Number of clusters & $3.7 \pm 1.6$ & $2.9 \pm 1.6$ & $2.9 \pm 1.6$ & $3.0 \pm 1.6$ & ns \\
\hline Number of switches & $5.8 \pm 3.8$ & $4.2 \pm 2.4$ & $4.2 \pm 2.4$ & $4.3 \pm 2.5$ & ns \\
\hline Total cluster size & $11.8 \pm 5.7$ & $6.9 \pm 4.3$ & $8.9 \pm 5.8$ & $8.6 \pm 5.1$ & $p<0.05$ \\
\hline Mean cluster size & $3.3 \pm 1.2$ & $2.2 \pm 0.8$ & $2.8 \pm 1.0$ & $2.8 \pm 1.0$ & $p<0.05$ \\
\hline Impaired performance, $n(\%)^{\mathrm{b}}$ & $9(31)$ & $8(57)$ & 7 (54) & $12(52)$ & $p<0.05$ \\
\hline \multicolumn{6}{|l|}{ Letter fluency } \\
\hline Number of words & $19.5 \pm 11.6$ & $22.4 \pm 11.3$ & $12.0 \pm 10.0$ & $14.6 \pm 9.7$ & ns \\
\hline Number of clusters & $5.4 \pm 3.8$ & $4.4 \pm 3.3$ & $2.9 \pm 2.9$ & $3.8 \pm 2.9$ & $p<0.05$ \\
\hline Number of switches & $14.1 \pm 9.6$ & $17.9 \pm 11.0$ & $6.3 \pm 5.0$ & $9.1 \pm 6.0$ & ns \\
\hline Total cluster size & $13.5 \pm 10.1$ & $9.7 \pm 6.9$ & $8.3 \pm 9.4$ & $9.0 \pm 7.2$ & $p<0.05$ \\
\hline Mean cluster size & $5.8 \pm 2.7$ & $4.9 \pm 2.5$ & $4.7 \pm 3.0$ & $5.1 \pm 2.4$ & ns \\
\hline Impaired performance, $n(\%)^{\mathrm{b}}$ & $8(30)$ & 3 (23) & $7(60)$ & $5(28)$ & ns \\
\hline
\end{tabular}

Data a mean \pm standard deviation unless otherwise specified. ns, not significant; bvFTD, behavioral variant frontotemporal dementia; PPA, primary progressive aphasia; SD, semantic dementia; PNFA, progressive nonfluent aphasia; LPA, logopenic progressive aphasia; MMSE, Mini-Mental State Examination. ${ }^{\mathrm{a}} p$ values refer to bvFTD vs. PPA. ${ }^{\mathrm{b}}$ Performance $<1$ st percentile (i.e., T-score $\leq 27$ ) compared to normative data.

Table 3. Single-measure intraclass correlations for the verbal fluency variables based on 2 raters

\begin{tabular}{lll}
\hline & Semantic fluency & Letter fluency \\
\hline Number of words & 1.0 & 0.99 \\
Number of clusters & 0.96 & 0.98 \\
Number of switches & 0.98 & 0.99 \\
Total cluster size & 0.98 & 0.97 \\
Mean cluster size & 0.90 & 0.80
\end{tabular}

\section{Baseline Verbal Fluency Performance}

In semantic fluency, $46 \%$ of the patients showed impaired performance compared with the normative data (i.e., a T-score $\leq 27$ ). Patients with PPA produced a significantly lower total number of words than patients with bvFTD $\left(F(1,75)=5.8, p<0.05, \eta^{2}=0.07\right)$. In addition, PPA patients produced smaller semantic clusters than patients with bvFTD (Table 2; total cluster size: $F(1,74)=6.3, \eta^{2}=0.08$; mean cluster size: $F(1,74)=6.3, p<0.05, \eta^{2}=0.08$ ). Post hoc comparisons within the PPA group showed no significant differences in the fluency variables between patients with semantic, patients with nonfluent/agrammatic, and patients with logopenic variants (all $p>0.05$ ). 
Fig. 1. Letter fluency performance in the primary progressive aphasia variant groups. ${ }^{*} p<0.05$. van den Berg et al.: Qualitative Assessment of Verbal Fluency Performance in Frontotemporal Dementia

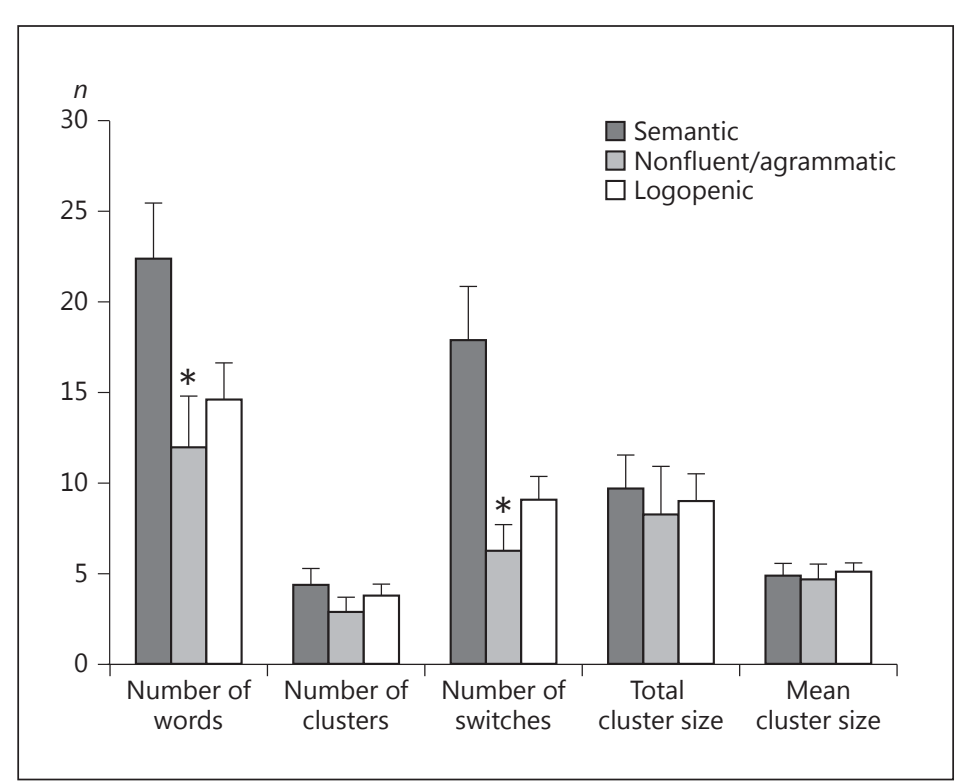

In letter fluency, $29 \%$ of the patients showed impaired performance compared with the normative data (i.e., a T-score $\leq 27$ ). The total number of words generated did not differ between patients with PPA and those with $\operatorname{bvFTD}\left(F(1,74)=1.5, p=0.22, \eta^{2}=0.02\right)$. However, patients with PPA produced a lower number of clusters and total cluster size than patients with bvFTD (Table 2; $F(1,70)=3.9, p<0.05, \eta^{2}=0.05 ; F(1,70)=4.1, p<0.05, \eta^{2}=0.06$ ). Post hoc comparisons within the PPA groups showed that patients with semantic variant PPA produced significantly more words $\left(F(2,43)=3.7, p<0.05, \eta^{2}=0.15\right)$ but also more switches $\left(F(2,43)=8.3, p<0.01, \eta^{2}=0.28\right.$, adjusted for total number of words) than patients with nonfluent/agrammatic and logopenic variants (Fig. 1).

\section{Change over Time in Verbal Fluency Performance}

Nineteen patients ( 3 bvFTD, 6 semantic, 4 nonfluent/agrammatic, and 6 logopenic) performed the semantic and letter fluency task at follow-up. For semantic fluency, the patient group as a whole showed a significant decline in total number of words $(F(1,18)=9.8, p<$ $\left.0.01, \eta^{2}=0.35\right)$ and cluster size $\left(F(1,18)=5.0, p<0.05, \eta^{2}=0.32\right)$. For letter fluency, the patient group as a whole showed a significant decline in total number of words $(F(1,18)=$ $\left.5.7, p<0.05, \eta^{2}=0.24\right)$, number of clusters $\left(F(1,18)=8.4, p<0.05, \eta^{2}=0.32\right)$, number of switches $\left(F(1,18)=10.3, p<0.01, \eta^{2}=0.37\right)$, and cluster size $\left(F(1,18)=7.3, p<0.05, \eta^{2}=\right.$ 0.29).

Examination of the time $\times$ group (bvFTD vs. PPA) interaction for semantic fluency showed that patients with PPA had a greater decline in total number of words $(F(1,17)=5.3, p<0.05$, $\left.\eta^{2}=0.24\right)$ and number of clusters $\left(F(1,17)=4.2, p<0.05, \eta^{2}=0.20\right)$ than patients with bvFTD. For letter fluency, no significant time $\times$ group interactions were observed.

\section{Association with Other Cognitive Functions}

The results of the regression analysis (Table 4) showed that all but one measure of semantic and letter fluency correlated significantly with information processing speed (range standardized regression coefficient $\beta: 0.14-0.54$ ). Furthermore, for semantic fluency the total number of words, the number of clusters, and the cluster size showed significant associations with the domain scores for language and memory ( $\beta$ 0.24-0.38). For letter fluency, the total 
van den Berg et al.: Qualitative Assessment of Verbal Fluency Performance in Frontotemporal Dementia

Table 4. Relation between measures of verbal fluency and cognitive domain scores

\begin{tabular}{llcll}
\hline & Language & Memory & $\begin{array}{l}\text { Processing } \\
\text { speed }\end{array}$ & $\begin{array}{l}\text { Attention and } \\
\text { executive functioning }\end{array}$ \\
\hline Semantic fluency & & & \\
$\quad$ Number of words & $0.35^{* *}$ & $0.24^{*}$ & $0.48^{* *}$ & $0.24^{*}$ \\
Number of clusters & $0.25^{*}$ & $0.25^{*}$ & $0.50^{* *}$ & 0.22 \\
Number of switches & 0.21 & -0.02 & $0.35^{*}$ & 0.22 \\
Total cluster size & $0.26^{*}$ & $0.38^{* *}$ & $0.47^{* *}$ & 0.17 \\
Mean cluster size & 0.07 & 0.26 & 0.14 & -0.04 \\
Letter fluency & & & & \\
Number of words & 0.14 & 0.03 & $0.55^{* *}$ & $0.35^{* *}$ \\
Number of clusters & 0.20 & 0.07 & $0.53^{* *}$ & 0.20 \\
Number of switches & 0.05 & -0.02 & $0.50^{* *}$ & $0.32^{*}$ \\
Total cluster size & 0.23 & 0.08 & $0.54^{* *}$ & 0.20 \\
Mean cluster size & $0.33^{*}$ & -0.04 & $0.50^{* *}$ & 0.11 \\
\hline
\end{tabular}

Data are age- and sex-adjusted standardized regression coefficient $\beta$ values. ${ }^{*} p<0.05,{ }^{* *} p<0.01$.

number of words and the number of switches correlated significantly with attention and executive functioning ( $\beta$ 0.32-0.35).

\section{Discussion}

The present study examined clustering and switching in verbal fluency in patients with bvFTD and PPA. We found both quantitative and qualitative differences in verbal fluency between these patient groups. Patients with PPA not only generated fewer words on semantic fluency than patients with bvFTD, but they also produced fewer and smaller clusters on semantic and letter fluency. These findings fit well with the theoretical notion that clustering relies on the generation of semantic, associative, or letter-related words and is thus mainly supported by the integrity of the semantic system. Damage to the left temporal lobe, as is present in PPA, evidently impairs access to lexical information and leads to deterioration of semantic storage [28].

Our results also showed differences between subtypes of PPA. Patients with the semantic variant produced more words than patients with the nonfluent/agrammatic and logopenic variants. The number of words generated in letter fluency in semantic variant PPA patients was comparable to that generated in the bvFTD group, reflecting the uncompromised fluency of speech in semantic variant PPA. However, semantic variant patients showed significantly more switches than patients with the nonfluent/agrammatic or logopenic variant. This relative increase in the number of switches in semantic variant PPA may reflect the combination of both intact fluency of speech and deterioration of semantic knowledge. Switching is thought to invoke a deliberate, controlled search and is associated with executive functioning [17]. It is mainly supported by frontal lobe functioning, which is relatively spared in semantic variant PPA, at least in the early stages of the disease.

As would be expected, patients with nonfluent/agrammatic PPA showed the lowest output in both semantic and letter fluency, which is thought to result primarily from speech apraxia [6, 8, 29-31]. The cluster sizes in patients with nonfluent/agrammatic PPA were, however, comparable to those found in patients with semantic and logopenic variant PPA. Whether the PPA subtypes might rely on different strategies to counter degradation of 
van den Berg et al.: Qualitative Assessment of Verbal Fluency Performance in Frontotemporal Dementia

semantic knowledge (e.g., spelling or sound similarities vs. semantic similarities) could not be derived from the present analysis. There were no clear differences in clustering and switching between patients with nonfluent/agrammatic and those with logopenic variant PPA.

The diagnostic utility of verbal fluency in identifying subtypes of dementia has been examined by several previous studies [7, 14, 30,32]. Although patients with FTD overall showed a worse fluency performance than patients with $\mathrm{AD}$, the disparity between letter and semantic fluency is particularly effective in differentiating the two patient groups [7]. Moreover, AD can be distinguished from nonfluent/agrammatic and semantic variant PPA based on their profile of verbal fluency (number of words, word frequency, and type of errors) [30] and a recent study indicated that measures of lexical similarity in verbal fluency predict conversion of MCI into AD [14]. In line with these findings, the results of the present study indicate that subtypes of FTD can also be distinguished by qualitative inspection of clustering and switching measures. More specifically, analysis of the clustering and switching variables supports the semantic deficit in semantic variant PPA in contrast to the agrammatism and motor speech errors in nonfluent/agrammatic PPA, thereby aiding timely and accurate diagnosis.

The scoring method used in the present study, as described by Ledoux et al. [17], is based on the original scoring by Troyer et al. [11]. The interrater reliability of this method was adequate in the present study (median 0.98) and highly similar to the interrater reliability reported in the original study by Ledoux et al. [17]. Clustering and switching are considered robust theory-based fluency measures, a notion that is supported by data from patients with schizophrenia, HIV, Huntington disease, Parkinson disease, multiple sclerosis, and traumatic brain injury $[12,13,33-36]$. A recent study showed that machine learning in verbal fluency outperformed traditional structural MRI measures in predicting conversion from $\mathrm{MCI}$ into $\mathrm{AD}$ (area under the curve for MRI measures 0.76, vs. 0.87 for machine learning predictors) [14]. Although this machine learning approach may not be easily adopted in standard clinical care, our findings provide further support for the diagnostic value of verbal fluency tests in FTD. Moreover, it might be useful to examine the value of different types of relationships (i.e., phonological, orthographic, or semantic) as these are not separately recorded in the Ledoux scoring method. In this regard, inclusion of an AD comparison group could potentially yield additional information on these processes. Longitudinal data on verbal fluency in relation to onset or progression of dementia are scarce. Raoux et al. [37] and Auriacombe et al. [38] showed that patients with AD already produce fewer words and switches 5 years before their clinical diagnosis. In addition, Pakhomov and Hemmy [39], using an automated approach, showed that in 239 cognitively intact subjects a larger semantic cluster size was associated with a 38 and $26 \%$ reduced dementia risk after 6 and 17 years, respectively. In the present study, clustering and switching in letter fluency showed a particularly strong decline in PPA patients, regardless of the subtype of PPA. Letter fluency thus appears to be a sensitive measure of cognitive decline in PPA.

Verbal fluency is supported by both common and distinct cognitive processes. Our results show a general association with measures of processing speed, which is in line with previous studies $[40,41]$ and probably reflects the timed nature of the task. Clustering in semantic fluency was significantly associated with memory and language, whereas in letter fluency switching was associated with executive functioning. These findings fit well within the theoretical model of verbal fluency proposed by Troyer et al. [11], in which clustering is thought to rely upon temporal lobe processes such as verbal memory and word storage, whereas switching relies upon frontal lobe processes such as strategic search processes, cognitive flexibility, and shifting.

Strengths of the present study include the relatively large patient sample and the detailed verbal fluency scoring method that showed a high interrater reliability. A limitation of this 
Dementia

Cognitive Disorders

\begin{tabular}{l|l}
\hline Dement Geriatr Cogn Disord 2017;44:35-44 \\
\hline DOI: 10.1159/000477538 & $\begin{array}{l}\text { C 2017 The Author(s). Published by S. Karger AG, Basel } \\
\text { www.karger.com/dem }\end{array}$ \\
\hline
\end{tabular}

van den Berg et al.: Qualitative Assessment of Verbal Fluency Performance in Frontotemporal Dementia

study is the fact that the fluency data in this study were part of the diagnostic neuropsychological assessment. Patients with worse performance in semantic fluency are more likely to be classified as having semantic variant PPA than nonfluent/agrammatic PPA. The clustering and switching measures were, however, specifically calculated for this study, and were therefore not used for the diagnostic workup. This study included patients with a clinical diagnosis of bvFTD or PPA; autopsy-confirmed cases were unfortunately unavailable. This means that no pathological confirmation of the dementia subtype was possible. In addition, some patients with a clinical diagnosis of logopenic variant PPA may have underlying Alzheimer-type pathology. Disease duration is a difficult measure to assess in FTD. We estimated disease duration as the time between the first report of symptoms and the clinical assessment. It is possible that bvFTD is diagnosed in a later stage than PPA as the presenting symptoms (behavioral changes vs. language impairment) are more easily recognized in PPA. Given that the patients with bvFTD tended to outperform the PPA patients on most fluency measures, this potential bias in the estimation of disease duration is unlikely to have significantly influenced our results.

In sum, the present study showed differences in clustering and switching in verbal fluency between patients with PPA and those with bvFTD and a significant decline in verbal fluency over time. Clustering was specifically associated with memory and language, and switching with executive functioning. These results show that qualitative differences in verbal fluency provide additional information on different cognitive functions in FTD and PPA that can be used in clinical practice to improve diagnostic accuracy.

\section{Disclosure Statement}

The authors declare that they have no conflict of interest.

\section{References}

1 Seelaar H, Rohrer JD, Pijnenburg YA, Fox NC, van Swieten JC: Clinical, genetic and pathological heterogeneity of frontotemporal dementia: a review. J Neurol Neurosurg Psychiatry 2011;82:476-486.

2 Rohrer JD, Rosen HJ: Neuroimaging in frontotemporal dementia. Int Rev Psychiatry 2013;25:221-229.

3 McKhann GM, Albert MS, Grossman M, Miller B, Dickson D, Trojanowski JQ: Clinical and pathological diagnosis of frontotemporal dementia: report of the Work Group on Frontotemporal Dementia and Pick's Disease. Arch Neurol 2001;58:1803-1809.

4 Grossman M: Frontotemporal dementia: a review. J Int Neuropsychol Soc 2002;8:566-583.

5 Rascovsky K, Hodges JR, Knopman D, Mendez MF, Kramer JH, Neuhaus J, van Swieten JC, Seelaar H, Dopper EG, Onyike CU, Hillis AE, Josephs KA, Boeve BF, Kertesz A, Seeley WW, Rankin KP, Johnson JK, Gorno-Tempini ML, Rosen H, Prioleau-Latham CE, Lee A, Kipps CM, Lillo P, Piguet O, Rohrer JD, Rossor MN, Warren JD, Fox NC, Galasko D, Salmon DP, Black SE, Mesulam M, Weintraub S, Dickerson BC, Diehl-Schmid J, Pasquier F, Deramecourt V, Lebert F, Pijnenburg Y, Chow TW, Manes F, Grafman J, Cappa SF, Freedman M, Grossman M, Miller BL: Sensitivity of revised diagnostic criteria for the behavioural variant of frontotemporal dementia. Brain 2011; 134(pt 9):2456-2477.

6 Gorno-Tempini ML, Hillis AE, Weintraub S, Kertesz A, Mendez M, Cappa SF, Ogar JM, Rohrer JD, Black S, Boeve BF, Manes F, Dronkers NF, Vandenberghe R, Rascovsky K, Patterson K, Miller BL, Knopman DS, Hodges JR, Mesulam MM, Grossman M: Classification of primary progressive aphasia and its variants. Neurology 2011; 76:1006-1014.

7 Rascovsky K, Salmon DP, Hansen LA, Thal LJ, Galasko D: Disparate letter and semantic category fluency deficits in autopsy-confirmed frontotemporal dementia and Alzheimer's disease. Neuropsychology 2007;21:20-30.

8 Libon DJ, McMillan C, Gunawardena D, Powers C, Massimo L, Khan A, Morgan B, Farag C, Richmond L, Weinstein J, Moore P, Coslett HB, Chatterjee A, Aguirre G, Grossman M: Neurocognitive contributions to verbal fluency deficits in frontotemporal lobar degeneration. Neurology 2009;73:535-542.

9 Cummings JL: The one-minute mental status examination. Neurology 2004;62:534-535.

10 Troyer AK, Moscovitch M, Winocur G, Alexander MP, Stuss D: Clustering and switching on verbal fluency: the effects of focal frontal- and temporal-lobe lesions. Neuropsychologia 1998;36:499-504. 
van den Berg et al.: Qualitative Assessment of Verbal Fluency Performance in

Frontotemporal Dementia

11 Troyer AK, Moscovitch M, Winocur G: Clustering and switching as two components of verbal fluency: evidence from younger and older healthy adults. J Clin Exp Neuropsychol 1997;11:138-146.

12 Zakzanis KK, McDonald K, Troyer AK: Component analysis of verbal fluency scores in severe traumatic brain injury. Brain Inj 2013;27:903-908.

13 Zakzanis KK, Troyer AK, Rich JB, Heinrichts W: Component analysis of verbal fluency in patients with schizophrenia. Neuropsychiatry Neuropsychol Behav Neurol 2000;13:239-245.

14 Clark DG, McLaughlin PM, Woo E, Hwang K, Hurtz S, Ramirez L, Eastman J, Dukes RM, Kapur P, DeRamus TP, Apostolova LG: Novel verbal fluency scores and structural brain imaging for prediction of cognitive outcome in mild cognitive impairment. Alzheimers Dement (Amst) 2016;2:113-122.

15 Verhage F: Intelligence and Age: Study with Dutch People Aged 12-77 (in Dutch). Assen, Van Gorcum, 1964.

16 Schmand B, Groenink SC, van den Dungen M: Letter fluency: psychometric properties and Dutch normative data (in Dutch). Tijdschr Gerontol Geriatr 2008;39:64-74.

17 Ledoux K, Vannorsdall TD, Pickett EJ, Bosley LV, Gordon B, Schretlen DJ: Capturing additional information about the organization of entries in the lexicon from verbal fluency productions. J Clin Exp Neuropsychol 2014;36:205-220.

18 Van der Elst W, van Boxtel MP, van Breukelen GJ, Jolles J: Rey's Verbal Learning Test: normative data for 1,855 healthy participants aged 24-81 years and the influence of age, sex, education, and mode of presentation. J Int Neuropsychol Soc 2005;11:290-302.

19 Wilson BA, Alderman N, Burgess PW, Emslie H, Evans JJ: Behavioural Assessment of the Dysexecutive Syndrome. Bury St. Edmunds, Thames Valley Test Company, 1996.

20 Lindeboom J, Schmand B, Tulner L, Walstra G, Jonker C: Visual association test to detect early dementia of the Alzheimer type. J Neurol Neurosurg Psychiatry 2002;73:126-133.

21 Kaplan E, Goodglass H, Weintraub S: The Boston Naming Test. Philadelphia, Lea \& Febiger, 1978.

22 Jolles J, Houx PJ, van Boxtel MPJ; RWHM Ponds: Maastricht Aging Study: Determinants of Cognitive Aging. Maastricht, Neuropsych Publishers, 1995.

23 Corrigan JD, Hinkeldey NS: Relationships between parts A and B of the Trail Making Test. J Clin Psychol 1987; 43:402-409.

24 Stroop JE: Studies of interference in serial verbal reactions. J Exp Psychol 1935;18:643-662.

25 Nelson HE: A modified card sorting test sensitive to frontal lobe defects. Cortex 1976;12:313-324.

26 Wechsler, D: Wechsler Adult Intelligence Scale, ed 3. San Antonio, Psychological Corporation, 1997.

27 Wilson BA, Cockburn J, Baddeley A: The Rivermead Behavioural Memory Test. Reading, Thames Valley Test Company, 1985.

28 Tröster AI, Fields JA, Testa JA, Paul RH, Blanco CR, Hames KA, Salmon DP, Beatty WW: Cortical and subcortical influences on clustering and switching in the performance of verbal fluency tasks. Neuropsychologia 1998;36: 295-304.

29 Rogers TT, Ivanoiu A, Patterson K, Hodges JR: Semantic memory in Alzheimer's disease and the frontotemporal dementias: a longitudinal study of 236 patients. Neuropsychology 2006;20:319-335.

30 Marczinski CA, Kertesz A: Category and letter fluency in semantic dementia, primary progressive aphasia, and Alzheimer's disease. Brain Lang 2006;97:258-265.

31 Mendez MF, Clark DG, Shapira JS, Cummings JL: Speech and language in progressive nonfluent aphasia compared with early Alzheimer's disease. Neurology 2003;61:1108-1113.

32 Rinehardt E, Eichstaedt K, Schinka JA, Loewenstein DA, Mattingly M, Fils J, Duara R, Schoenberg MR: Verbal fluency patterns in mild cognitive impairment and Alzheimer's disease. Dement Geriatr Cogn Disord 2014;38: $1-9$.

33 Troyer AK, Moscovitch M, Winocur G, Leach L, Freedman M: Clustering and switching on verbal fluency tests in Alzheimer's and Parkinson's disease. J Int Neuropsychol Soc 1998;4:137-143.

34 Iudicello JE, Woods SP, Weber E, Dawson MS, Scott JC, Carey CL, Grant I: Cognitive mechanisms of switching in HIV-associated category fluency deficits. J Clin Exp Neuropsychol 2008;30:797-804.

35 Ho AK, Sahakian BJ, Robbins TW, Barker RA, Hodges JR: Verbal fluency in Huntington's disease: a longitudinal analysis of phonemic and semantic clustering and switching. Neuropsychologia 2002;40:1277-1284.

36 Messinis L, Kosmidis MH, Vlahou C, Malegiannaki AC, Gatzounis G, Dimisianos N, Karra A, Kiosseoglou G, Gourzis P, Papathanasopoulos P: Phonological fluency strategy of switching differentiates relapsing-remitting and secondary progressive multiple sclerosis patients. ISRN Neurol 2013;2013:451429.

37 Raoux N, Amieva H, Le Goff M, Auriacombe S, Carcaillon L, Letenneur L, Dartigues JF: Clustering and switching processes in semantic verbal fluency in the course of Alzheimer's disease subjects: results from the PAQUID longitudinal study. Cortex 2008;44:1188-1196.

38 Auriacombe S, Lechevallier N, Amieva H, Harston S, Raoux N, Dartigues J-F: A longitudinal study of quantitative and qualitative features of category verbal fluency in incident Alzheimer's disease subjects: results from the PAQUID study. Dement Geriatr Cogn Disord 2006;21:260-266.

39 Pakhomov SV, Hemmy LS: A computational linguistic measure of clustering behavior on semantic verbal fluency task predicts risk of future dementia in the nun study. Cortex 2014;55:97-106.

40 Abwender DA, Swan JG, Bowerman JT, Connolly SW: Qualitative analysis of verbal fluency output: review and comparison of several scoring methods. Assessment 2001;8:323-338.

41 Mayr U: On the dissociation between clustering and switching in verbal fluency: comment on Troyer, Moscovitch, Winocur, Alexander and Stuss. Neuropsychologia 2002;40:562-566. 